\title{
Modulator treatment for people with cystic fibrosis: moving in the right direction
}

\author{
J. Stuart Elborn
}

Affiliation: Faculty of Medicine Health and Life Sciences, Queens University of Belfast, Belfast, UK.

Correspondence: J. Stuart Elborn. Faculty of Medicine Health and Life Sciences, Queens University of Belfast, 90 Lisburn Rd, Belfast, BT9 6AG, UK. E-mail: s.elbornaqub.ac.uk

@ERSpublications

As delivery of $\mathrm{CF}$ healthcare services evolves, it is important for respiratory clinical teams and the wider group of specialists in the MDT to understand how modulator drugs are used, the side-effects and potential for drug interactions http://bit.ly/3akQ0tK

Cite this article as: Elborn JS. Modulator treatment for people with cystic fibrosis: moving in the right direction. Eur Respir Rev 2020; 29: 200051 [https://doi.org/10.1183/16000617.0051-2020].

In the past 10 years the incremental success of modulators of the cystic fibrosis transmembrane regulator (CFTR) protein have improved outcomes for people with cystic fibrosis (CF) [1]. Ivacaftor for individuals with sequence variants associated with class III mutations (e.g. G551D) and some other residual function mutations of the CFTR gene followed by lumacaftor/ivacaftor or tezacaftor/ivacaftor combinations in individuals homozygous for the F508del have achieved improvements in quality of life reduction, pulmonary exacerbations and variable improvement in forced expiratory volume in $1 \mathrm{~s}\left(\mathrm{FEV}_{1}\right)$. Highly effective modulator treatment, a triple combination of tezacaftor/ivacaftor and elexacaftor (a second modulator with the CFTR potentiator), was granted market approval in October 2019 by the US Food and Drug Administration. This approval is "for patients 12 years and older with CF who have at least one F508del mutation in the CFTR gene, which is estimated to represent $90 \%$ of the CF population" [2].

These drugs are progressively transforming the quality of life of people with $\mathrm{CF}$ and demonstrate improvements in important surrogates of survival, $\mathrm{FEV}_{1}$ and pulmonary exacerbation frequency [3]. There is also a useful biomarker of response, sweat chloride concentrations, which is useful in stratified groups but less helpful in predicting individual responses. Highly effective modulator treatment is currently being assessed by the European Medicines Agency with an anticipated decision expected in the last 6 months of 2020. The results from the clinical trials of this triple therapy demonstrate even greater improvements in lung function and quality of life with a significant reduction in pulmonary exacerbations compared to the two dual therapies of lumacaftor/ivacaftor and tezacaftor/ivacaftor. Therefore, it would be surprising if approval is not granted for the European market, though the European Medicines Agency's considerations will determine which patient groups this new treatment is approved for. It is hoped that these will closely align with the approval from the US Food and Drug Administration. For the USA, this may result in $>85-$ 90\% of people in Europe with CF being on highly effective modulator therapy with consequent improvement in quality of life and reduced requirement for hospital admission.

In this issue of the European Respiratory Review, SHTEInBERg and TAYLOR-Cousar [4] provide a very useful review of the current state of play in Europe for CFTR modulators [5]. This article helpfully puts in context current treatment with modulators in Europe and other countries outside the USA while we await

Received: 24 Feb 2020 | Accepted after revision: 27 Feb 2020

Provenance: Commissioned article, peer reviewed.

Copyright $\odot$ ERS 2020. This article is open access and distributed under the terms of the Creative Commons Attribution Non-Commercial Licence 4.0. 
the more effective triple combination [4]. The dual therapy and indeed ivacaftor are not available in all countries where $\mathrm{CF}$ is a common. The current report provides the evidence base for improvements in $\mathrm{FEV}_{1}$, quality of life and the reduction in pulmonary exacerbations following treatment with ivacaftor and dual therapy. Ivacaftor and the two combination therapies of lumacaftor or tezacaftor plus ivacaftor improve $\mathrm{FEV}_{1}$ by around $3 \%$. This effect is much greater (5-10\%) in people with CF who have class III or residual function mutations with ivacaftor treatment compared to individuals who are homozygous for the for the F508del mutation and receive the dual combination therapies. In their synthesis of the data from clinical trials and real-world usage, SHTEINBERg and TAYLOR-Cousar [4] draw some important conclusions. There is a benefit from these therapies even in individuals with severely reduced $\mathrm{FEV}_{1}$ who are excluded from the trials in follow-up clinical studies, and people with CF benefit from a significant reduction in pulmonary exacerbations. This is usually associated with a modest improvement in $\mathrm{FEV}_{1}$. The explanation for this is that in severe lung disease there is little room to improve spirometry but reducing the mucus burden still results in a benefit by reducing pulmonary exacerbations. It is interesting that he $\mathrm{FEV}_{1}$ response does not predict the impact on exacerbations, suggesting additional benefits of modulator therapy in people with severe disease on top of reducing airflow obstruction.

The adverse events and drug/drug interactions are comprehensively covered in the article by SHTEINBERg and TAYLOR-COUSAR [4]. The combination of lumacaftor/ivacaftor has the unpleasant side-effect of a strong sensation of chest tightness and often a reduction in $\mathrm{FEV}_{1}$. This occurs in up to $20 \%$ of patients and is more common in those with a lower $\mathrm{FEV}_{1}$. On occasions this has required the drug to be discontinued and there is no agreed mechanism which explains this. Many clinicians have used complex regimes introducing lumacaftor/ivacaftor gradually often during a hospital admission to manage this problem.

Drug/drug interactions are also important in this group of drugs as they interact with a number of hepatic enzymes important in drug metabolism. These are particularly relevant for antibacterial and antifungal agents, which are often used in people with CF. Additional issues for oral contraceptive and anticoagulant use are also highlighted. These interactions are important as people with CF interact with a wide range of clinical disciplines, including primary care. This will increase in the future as adults with CF live longer and are in better health and may have more interaction with clinical teams in addition to the CF team.

The delivery of healthcare services for people with CF is likely to evolve over the next decade with a wide implementation of highly effective modulator treatment. It is important for respiratory clinical teams and the wider group of specialists in the multidisciplinary team to understand how these drugs are used, the side-effects and potential for drug interactions. The article by SHTEInBerg and TAYlor-Cousar [4] provides a very useful summary of the current position.

Conflict of interest: J.S. Elborn has nothing to disclose.

\section{References}

$1 \quad$ Elborn JS. Cystic fibrosis. Lancet 2016; 388: 2519-2531.

2 FDA news release. FDA approves new breakthrough therapy for cystic fibrosis. www.fda.gov/news-events/ press-announcements/fda-approves-new-breakthrough-therapy-cystic-fibrosis Date last updated: 21 October 2019.

3 Elborn JS. Adult care in cystic fibrosis. Semin Respir Crit Care Med 2019; 40: 857-868.

4 Shteinberg M, Taylor-Cousar JL. Impact of CFTR modulator use on outcomes in people with severe cystic fibrosis lung disease. Eur Respir Rev 2020; 29: 190112.

5 McColley SA, Konstan MW, Ramsey BW, et al. Lumacaftor/ivacaftor reduces pulmonary exacerbations in patients irrespective of initial changes in FEV(1). J Cyst Fibros 2019; 18: 94-101. 